\title{
PREHYPERTENSION: DOES IT REALLY MATTER?
}

The arterial blood pressure (AP) is an integral index reflecting the functional state of the cardiovascular system. Instead of it being a dynamic parameter with short- term and circadian deviations, it is maintained in definite referent values. The argument of the European Society of Cardiology (ESC) to define AP values under $140 / 90 \mathrm{mmHg}$ in three categories - optimal (under 120/80 mmHg), normal (120-129 mmHg for systolic and/ or $80-84 \mathrm{mmHg}$ for diastolic) and high normal (130-139 $\mathrm{mmHg}$ for systolic and/or $85-89 \mathrm{mmHg}$ for diastolic) is connected to the different cardiovascular risks. The risk of arterial hypertension (AH) to develop is higher in individuals with high normal AP. In prehypertension, the systolic (top number) reading is $120 \mathrm{mmHg}-139$ $\mathrm{mmHg}$, or the diastolic (bottom number) reading is $80 \mathrm{mmHg}-89 \mathrm{mmHg}$.

Prehypertension is a warning sign that the individual may get high blood pressure in the future. High blood pressure increases the risk of heart attack, stroke, coronary heart disease, heart failure, and kidney failure. There is no cure for high blood pressure, but there is treatment with diet, lifestyle habits, and medications. The individuals with HNAP are of interest because of the possibility for them to be treated by a non-drug therapy and thus delaying the $\mathrm{AH}$ appearance and reducing the cardiovascular risk.

Julia Nikolova et al. made an observation study in two groups: with HNAP and controls with optimal AP. The objective of the study is for the risky profile and some haemodynamic indices in the students of medicine to be followed.

Students of medicine are a special group, taking into consideration their non-dynamics and proclivity for obesity, predisposing them thus to AH. The hemodynamic profile of HNAP students of medicine indicates increased cardiac frequency and shortened duration for the left ventricular ejection and could be connected to increased sympathetic nerve activity.

As far as the HNAP individuals' therapeutic plan is concerned, the recommendation is directed to risk profile correction - reduction of body weight, consumption of salt, no smoking. The increased physical activity could regulate the autonomic nerve system disorder and reduce the sympathetic tone activity. The early risk factors correction in students of medicine will delay the appearance of $\mathrm{AH}$ and will reduce the possibility of the appearance of cardiovascular incidents.

Petar Nikolov et al. analyzed Intima media thickness (IMT), Ankle Brachial Index (ABI) and FlowMediated Dilation (FMD) in 103 individuals with pre-hypertension and 45 normotensives. The first two indices reflect structural arterial vascular alterations, while FMD is referred to endothelial changes. FMD is impaired in $\mathrm{PH}$ and correlates with the cardiovascular risk factors and diseases. The decreased percentage of FMD is connected to the vasodilators/vasoconstrictors ratio shifted to the right. In their study, FMD is significantly lower in pre-hypertensives. It is a good predictor for vascular impairment and may serve in determining the therapeutic approach.

In contrast to the FMD data, there are no differences in the IMT and the ABI indices in PH individuals compared to the normotensives ones. The last two indices are a sign of advanced atherosclerotic process. This proves that $\mathrm{PH}$ is a state of functional changes, yet in time it leads to structural cardiovascular alterations and the appearance of hypertension.

Prof. Snezhana Tisheva, MD, PhD, DMSc, FESC Medical University of Pleven 